\title{
Searching for massive star magnetospheres $\dagger$
}

\section{David Bohlender and Dmitry Monin}

\author{
Herzberg Institute of Astrophysics, National Research Council of Canada, \\ 5071 West Saanich Road, Victoria BC, Canada V9E 2E7
}

\begin{abstract}
We review the status of a long-term program to search for stellar magnetospheres in Bp stars. A few new $\sigma$ Ori $\mathrm{E}$ analogues discovered during the course of this investigation are briefly discussed and other stars that may be worthy of further study are noted.
\end{abstract}

Keywords. stars: chemically peculiar, stars: circumstellar matter, stars: early-type, stars: emission-line, stars: magnetic fields, stars: individual ( $\sigma$ Ori E, HD 176582)

\section{Introduction}

Most magnetic Bp stars that show evidence for magnetospheric material in the form of variable $\mathrm{H} \alpha$ emission or shell absorption and satellite UV lines are also non-thermal radio sources. The primary example is the prototypical helium-strong star $\sigma$ Ori E. Because of this, we have been conducting a long-term program on the Dominion Astrophysical Observatory's (DAO) 1.2-m and 1.8-m telescopes to search for variable $\mathrm{H} \alpha$ profiles and magnetic fields in other northern Bp stars known to be radio sources. Table 1 lists our current program objects, selected from compilations of radio emission surveys of Ap and Bp stars by Linsky et al. (1992), Leone et al. (1994) and Drake et al. (2006).

Table 1. Bright Bp stars with non-thermal radio emission.

\begin{tabular}{|c|c|c|c|c|c|c|c|}
\hline HD & Name & $\begin{array}{c}\text { Spectral } \\
\text { Type }\end{array}$ & $\begin{array}{c}v \text { sin } i \\
\left(\mathbf{k m ~ s} \mathbf{s}^{-1}\right.\end{array}$ & $\begin{array}{c}\mathbf{6} \mathbf{c m} \text { Flux } \\
\text { (mJy) }\end{array}$ & $\begin{array}{c}\mathbf{B}_{e} \text { Field } \\
\text { Extrema (kG) }\end{array}$ & $\begin{array}{c}\text { H } \alpha \\
\text { Variability }{ }^{1}\end{array}$ & $\begin{array}{c}\text { Period } \\
\text { (d) }\end{array}$ \\
\hline 12447 & $\alpha$ Psc & A2 SiSrCr & 75 & $0.36 \pm 0.09$ & -0.5 to 0.4 & $?$ & 1.491 \\
19832 & 56 Ari & B8 Si & 80 & $0.45 \pm 0.12$ & -0.4 to 0.4 & No & 0.72790 \\
35298 & V1156 Ori & B6 He-wk & $60 ?$ & $0.29 \pm 0.10$ & -4.5 to 4.5 & $?$ & 1.8111 \\
35502 & & B6 SrCrSi & $80 ?$ & $2.97 \pm 0.10$ & -3.0 to 0.0 & Emission & $0.8538 ?$ \\
36313 & V1093 Ori & B8 He-wk Si & 35 & $0.49 \pm 0.06$ & -1.5 to -1.1 & SB2? & $0.592 ?$ \\
36485 & O Ori C & B3 He-str & 32 & $0.95 \pm 0.07$ & -3.0 to -1.7 & Emission & 1.47775 \\
37150 & HR 1906 & B3V & 190 & $0.51 \pm 0.07$ & $\leqslant 0.5$ & $?$ & $?$ \\
37642 & V1148 Ori & B9 He-wk Si & 100 & $0.60 \pm 0.07$ & -3 to 2.7 & $?$ & $1.0787 ?$ \\
79158 & 36 Lyn & B9 He-wk & 48 & $0.45 \pm 0.05$ & -1.5 to 1.0 & Shell episodes & 3.83475 \\
124224 & CU Vir & B9 Si & 130 & $4.07 \pm 0.14$ & -1.0 to 1.0 & No & 0.5207 \\
164429 & HR 6718 & B9 SiCrSr & $95 ?$ & $0.30 \pm 0.05$ & -0.8 to 0.8 & $?$ & 1.0820 \\
170000 & $\phi$ Dra & A0 Si & 75 & $0.45 \pm 0.05$ & -0.2 to 0.6 & $?$ & 1.7165 \\
171247 & HR 6967 & B8 Si & 60 & $3.04 \pm 0.09$ & 0.0 to $1.2 ?$ & $?$ & 3.91227 \\
176582 & HR 7185 & B5 He-wk & 100 & $0.46 \pm 0.05$ & -2.0 to 2.0 & Emission & 1.58199 \\
196178 & HR 7870 & B8 Si & 50 & $3.00 \pm 0.07$ & -1.4 to -0.4 & $?$ & $\approx 10 ?$ \\
\hline
\end{tabular}

${ }^{1}$ A question mark indicates that phase coverage is insufficient to draw a conclusion about $\mathrm{H} \alpha$ variability.

\section{Results}

We first discovered variable $\mathrm{H} \alpha$ emission with a 1.47775 day period in the heliumstrong star $\delta$ OriC (Leone et al. 2010). While its emission variability is quite similar to that of $\sigma$ Ori E, in contrast to the prototypical helium-strong star, $\delta$ Ori C's low $v \sin i$

$\dagger$ Based on observations acquired at the Dominion Astrophysical Observatory, Herzberg Institute of Astrophysics, National Research Council of Canada. 

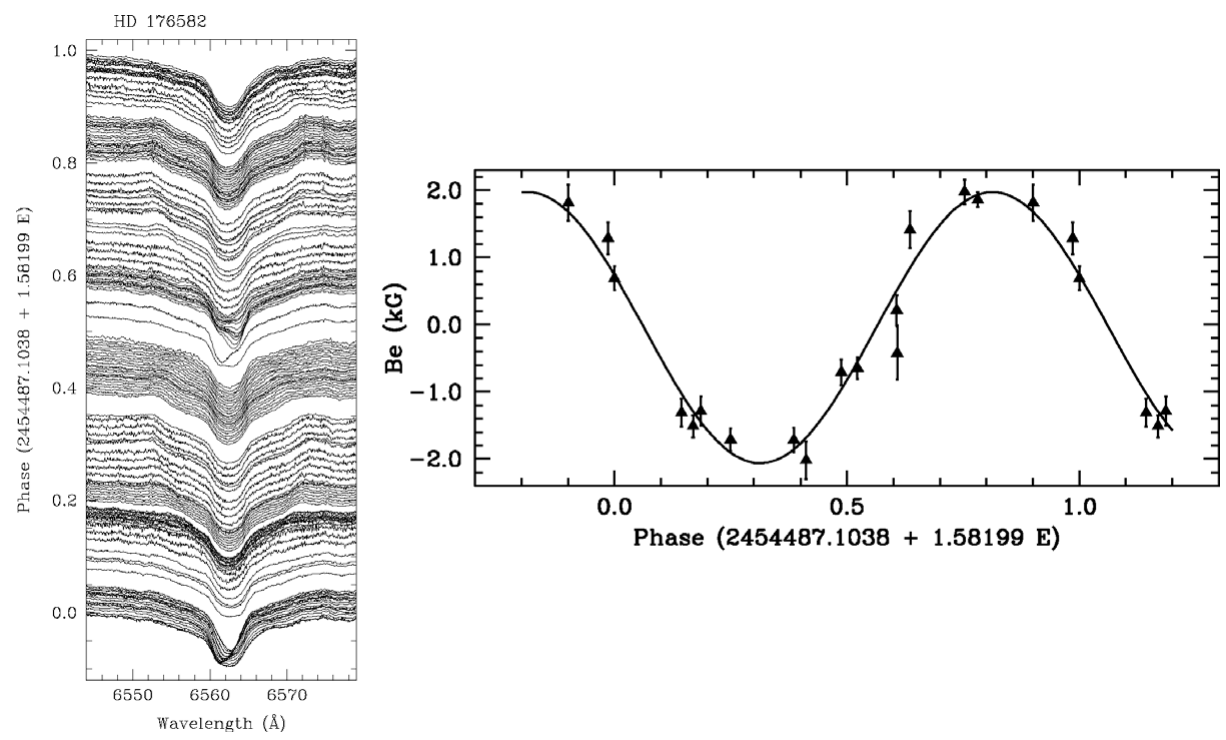

Figure 1. $\mathrm{H} \alpha$ emission and magnetic field variability of HD 176582. Note that shell absorption episodes occur when the magnetic equator crosses the line of sight (near phases 0.0 and 0.5 ).

and approximately constant $-2.5 \mathrm{kG}$ magnetic field show that it has a small inclination and a relatively small magnetic obliquity.

The helium-weak star 36 Lyn shows less obvious evidence of magnetospheric material. Two brief "shell" episodes occur for about $10 \%$ of its rotation period just as the magnetic equator crosses the line of sight to the observer (Wade et al. 2006; Smith et al. 2006).

The magnetic helium-weak star HD 35502 also has variable $\mathrm{H} \alpha$ emission. DAO magnetic field measurements suggest that it has a strong, non-reversing magnetic field with $\mathrm{a}-3 \mathrm{kG}$ extremum. The star is a rapid rotator and we are continuing to acquire data to try to establish a unique rotation period among several possibilities near 0.85 days.

We have recently discovered that the helium-weak star HD 176582 is also a magnetic $\mathrm{H} \alpha$ emission variable. DAO polarimeter data show a strong, approximately dipolar magnetic field with field extrema of $\pm 2 \mathrm{kG}$. Figure 1 shows our phase-resolved $\mathrm{H} \alpha$ and magnetic field data; the $\mathrm{H} \alpha$ variability is weaker but very similar in appearance to that of $\sigma$ Ori E.

\section{Conclusion}

A few other stars in Table 1 warrant additional observations. Our DAO magnetic field observations of HD 35298 show that it has a very strong $\approx 5 \mathrm{kG}$ field but our small set of $\mathrm{H} \alpha$ spectra for the star do not sample its rotation period very well. We have also detected magnetic fields in the non-thermal radio sources HD 164429 and HD 171247.

\section{References}

Drake, S. A., Wade, G. A., \& Linsky, J. L. 2006, in: A. Wilson (eds.), The X-ray Universe 2005, ESA-SP 604, p. 73

Leone, F., Trigilio, C., \& Umana, G. 1994, A\&AA, 283, 908

Leone, F., Bohlender, D. A., Bolton, C. T., Buemi, C. et al. 2010, MNRAS, 401, 2739

Linsky, J. L., Drake, S. A., \& Bastian, T. S. 1992, ApJ, 393, 341

Smith, M. A., Wade, G. A., Bohlender, D. A., \& Bolton, C. T. 2006, A\&\&A, 458, 581

Wade, G. A., Smith, M. A., Bohlender, D. A., Ryabchikova, T. A. et al. 2006, A\&A, 458, 569 